\title{
PROLONGATIONS AND COMPLETIONS OF RIEMANNIAN MANIFOLDS
}

\author{
STEPHANIE ALEXANDER \& RICHARD L. BISHOP
}

A Riemannian prolongation of a connected Riemannian manifold $M$ is a connected Riemannian manifold $N$ of the same dimension, along with a proper isometric imbedding of $M$ in $N$. If $N$ is complete, the prolongation is called a Riemannian completion.

In the first section, we give some examples and formulate some obstructions to prolongation at a point of the Cauchy boundary of $M$. The examples show that there may not exist maximal subsets of the Cauchy boundary on which $M$ can be prolonged, and that $M$ may admit a prolongation on its whole Cauchy boundary whithout admitting a Riemannian completion. The main theorem of $\S 2$ states that for any Riemannian manifold there is a nonprolongable metric with the same Cauchy sequences in the class of conformally equivalent metrics. In $\S 3$, a theorem for conformally equivalent complete metrics is proved, which gives a sufficient condition for the existence of Riemannian completions.

\section{Prolongations}

A Riemannian prolongation determines a metric prolongation of the underlying metric space, and a Riemannian completion determines, by the HopfRinow theorem, a metric completion. Specifically, by a metric prolongation of a metric space $M$ we mean a metric space $N$ and a proper distance-nonincreasing injection of $M$ in $N$, with $N$ complete giving a metric completion. For example, the Cauchy completion $C M$ consists of equivalence classes under asymptotism of Cauchy sequences in $M$, with the obvious metric and embedding. We denote by $\dot{M}$ the Cauchy boundary $C M-M$. A metric prolongation of $M$ to $N$ is said to prolong on a subset $S$ of $\dot{M}$ if the Cauchy sequences which determine $S$ converge in $N$. Note that we do not require the corresponding map of $S$ into $N$ to be injective; indeed, for Riemannian prolongations the geometry of $M$ may force identifications on $\dot{M}$ (see Example 1). $S$ will be called a prolongation set; the prolongation set of the prolongation will be the largest such $S$.

From now on, prolongation will mean Riemannian prolongation. We can make several simple observations. For a Riemannian manifold, $C M$ need not

Received August 8, 1971. 
be a topological manifold with boundary, even if $M$ admits a prolongation at each point of $\dot{M}$ (Examples 2, 3). However, it follows from the definition (by arclength) of distance in $M$ that any open $d_{C M}$-ball centered at a point of $\dot{M}$ intersects $M$ in a connected set, where $d_{C M}$ denotes distance in $C M$. Since the closure of a connected set is connected, this shows that $C M$ is locally connected. As in the examples, $\dot{M}$ does not have to be locally connected. The prolongation set of a prolongation of $M$ is open in $\dot{M}$. This is because if $N$ prolongs $M$ at $p \in \dot{M}$, then $N$ contains a compact $d_{N}$-ball of some radius $c>0$ about the image of $p$; points of $\dot{M}$ having $C M$-distance less than $c$ from $p$ are represented by $d_{M}$-Cauchy sequences which fall in $B$ and hence converge in $N$.

We mention some related papers. It has been observed by Bochner [1] and Dubois [2] that the relation " $N$ is a prolongation of $M$ " is an inductive partial ordering, and consequently there are maximal prolongations. A complete Riemannian manifold is nonprolongable but the converse is not true, according to examples due to Bochner. In the real analytic case, prolongations have been studied under the name continuations. Specifically, Rinow [6] and Myers [4] showed that a simply connected complete continuation is unique.

Some useful examples can be constructed from the Euclidean plane $P$ with the usual metric:

Example 1. Let $M$ be the simply connected covering of the space obtained by removing from $P$ a closed segment of length $1 . \dot{M}$ is a chain of countably many copies of the removed segment joined end to end, and $\dot{M}$ with the induced metric is isometric to $\boldsymbol{R}$. We choose an isometry $\varphi: \boldsymbol{R} \rightarrow \dot{M}$ for which the joining points of the chain are integers. If $M$ is prolonged on $\varphi(I)$ for some interval $I$, then $I$ cannot contain more than one integer. For example, suppose $0 \in I$. If $x \in I$ and $|x|<1$, then $M$ is prolonged at $\varphi(-x)$ as well as at $\varphi(x)$ and the two are identified, since in the prolonging space they both lie on the same geodesic ray $\gamma$ starting at $\varphi(0)$. The prolongation cannot extend to either $\varphi(1)$ or $\varphi(-1)$ because then it would extend to both, and $\gamma$ would have two distinct geodesic extensions on different sheets above $P$. Thus the prolongation set of any prolongation of $M$ has the form: an open subset of $\boldsymbol{R}$ for which each component includes at most one integer and for which any component including an integer is symmetric about it. Conversely, $M$ can be prolonged on any set of this form. In particular, in this example the union of nested prolongation sets is a prolongation set, so prolongation sets lie in maximal ones. However, we see that even in the analytic case, the union of prolongation sets need not be one again.

Example 2. Let $l_{k}$, for any nonzero integer $k$, be the closed segment in $P$ joining the origin to $q(k)=(\cos \pi /(2 k), \sin \pi /(2 k))$, and let $l_{\infty}$ be the closed segment joining the origin to $(1,0)$. Choose a sequence $\left\{p_{i}\right\}(i=1,2, \ldots)$ in $P$ converging to the origin, and such that $p_{i}$ lies in the open wedge bounded by $l_{i}$ and $l_{i+1}$. About each $p_{i}$ choose an open ball with closure in that open wedge. Construct $M$ from $P$ by removing $l_{\infty}$ and all the $l_{k}, k= \pm 1, \pm 2, \cdots$, and 
altering the metric within the small balls so that the curvatures at the $p_{i}$ diverge to $\infty$. The latter can be done by a conformal change of metric which decreases distances, but never by more than a factor of $1 / 2$, as in Theorem 1 below.

If $\dot{M}^{\prime}$ denotes $\dot{M}$ with the point $q$ corresponding to $(1,0)$ removed, then $\dot{M}^{\prime}$ is a chain of double copies of the $l_{k}$ and is homeomorphic to $\boldsymbol{R} . \dot{M}$ itself is connected, but not locally connected at $q$, and is not compact.

Let $S=\dot{M}^{\prime}-\{q(i) \mid i>0\}$. We claim that the prolongation sets which include $S$ are just the sets obtained from $\dot{M}^{\prime}$ by deleting any infinite subsequence $\{q(i(n))\}$. To construct a prolongation of $M$ on such a set, one must glue back together the two copies of $l_{k}$ for each $k$ not in $\{i(n)\}$. Then borders, disjoint from $M$ and each other, can be added to the wedges bounded by $l_{i(n)}$ and $l_{i(n+1)}$. On the other hand, if only finitely many $q(i)$ are excluded, the reidentification of the two copies of the $l_{i}$ makes $\left\{p_{i}\right\}$ into a convergent sequence, which is impossible. As a consequence, $S$ is not contained in any maximal prolongation set.

Example 3. The same construction as in Example 2, except that $\left\{p_{i}\right\}$ is chosen to converge to $(c, 0), 0<c<1$, instead of to the origin. Then the description of $\dot{M}$ remains unchanged. However, now $M$ may be prolonged on $\dot{M}$, in particular by replacing $l_{k}$ and $l_{\infty}-\{(c, 0)\}$. $M$ does not admit a Riemannian completion, since a completion would replace the $l_{k}$ and all of $l_{\infty}$.

We can spell out some obstructions to prolonging $M$ at a point $p \in \dot{M}$ by negating standard properties of the supposed prolongation $N$ and stating these properties in terms of $M$. In the routine examples which we are familiar with, every point on which there is no prolongation possesses one of these obstructions. We think there are other obstructions independent of these. Moreover, we would like to have obstructions to prolongation on a larger subset $S \subset \dot{M}$. (Note that Examples 1, 2 are prolongable on every point of $\dot{M}$.)

Curvature obstructions. These are based on the fact that the sectional curvature is a continuous function on the bundle of plane sections over $N$. Thus we need a way way of deciding when a sequence of sections on $M$ should converge in $N$. However, the bundle of plane sections is provided with a natural Riemannian metric for which the projection onto $M$ is a Riemannian submersion and the fibers are isometric to the Grassmannian symmetric space. The horizontal lifts of curves in $M$ with respect to the submersion structure are simply the parallel translates of plane sections along the curves. By using this parallel translation along a curve converging to a point of $\dot{M}$ the Cauchy completion of the bundle becomes a bundle over $C M$. With these remarks the following becomes sensible and obvious.

Proposition 1. (a) Let $\left\{\pi_{i}\right\}$ be a Cauchy sequence of plane sections of $M$ with base sequence convergent to $p \in \dot{M}$. If $\lim K\left(\pi_{i}\right)$ does not exist, then there is no prolongation on $p$.

(b) If there is any sequence of plane sections $\left\{\pi_{i}\right\}$ with base sequence 
convergent to $p \in \dot{M}$ and $K\left(\pi_{i}\right)$ unbounded, then there is no prolongation on $p$.

Convexity obstructions. A prolongation $N$ contains convex spherical neighborhoods centered at $p$. This immediately gives us the following.

Proposition 2. If in every spherical neighborhood in CM with center $p \in \dot{M}$ there are pairs of points in $M$ which are connected by more than one geodesic segment in that neighborhood, then there is no prolongation on $p$.

Solid angle obstructions. A small sphere in $N$ centered at $p$ has a Riemannian volume which approximates the volume of a sphere of the same radius in Euclidean space. Let $S_{r}(p)=\{q \in M \mid$ there is a geodesic segment of length $r$ from $q$ to $p$ \}. For sufficiently small $r$ this must be a subset of the sphere of radius $r$ and center $p$ in $N$. Let $d=\operatorname{dimension} M$, and $\Omega_{d-1}=$ the $(d-1)$ dimensional measure of a unit sphere in $E^{d}$.

Proposition 3. Under either of the following conditions there is no prolongation on $p \in \dot{M}$.

(a) For sufficiently small $r, S_{r}(p)$ has $(d-1)$-dimensional measure $\mu\left(S_{r}(p)\right)$ which satisfies

$$
\lim _{r \rightarrow 0} \mu\left(S_{r}(p)\right) / r^{d-1}>\Omega_{d-1}
$$

(b) For sufficiently small $r, S_{r}(p)$ is diffeomorphic to a $(d-1)$-sphere and

$$
\lim _{r \rightarrow 0} \mu\left(S_{r}(p)\right) / r^{d-1}<\Omega_{d-1}
$$

Examples. A cone, for which $\dot{M}$ consists of only the vertex, exhibits the convexity obstruction and the solid angle obstruction (b). Any proper covering space of the punctured plane has a single boundary point which illustrates the solid angle obstruction (a). The metric $x\left(d x^{2}+d y^{2}\right)$ on the half-plane $x>0$ has all three kinds of obstructions at its single boundary point.

\section{Nonprolongable metrics}

The following lemmas examine the effect of certain conformal changes in a metric. The results are local, so we must understand them as applying only to a sufficiently small neighborhood of $p_{0}$.

Lemma 1. Let $M$ be a Riemannian manifold with metric $\langle\rangle,, p_{0} \in M$, $r: M \rightarrow \boldsymbol{R}$ the distance from $p_{0}$. Let $\varphi: \boldsymbol{R} \rightarrow \boldsymbol{R}$ be a positive $C^{\infty}$ function. Then the geodesics through $p_{0}$ are also pregeodesics for the metric $\varphi\left(r^{2}\right)\langle$,$\rangle .$

Proof. Except for tangent vectors at $p_{0}$, we can decompose a tangent vector into its components in the direction of the geodesic from $p_{0}$, the radial component, and the orthogonal complement, the normal component. Since the new metric is conformal to the old, this decomposition is the same with respect to either (always using the geodesics of the old metric). We shall show that radial geodesics have the length-minimizing property for the new metric.

If $\gamma$ is a curve from $p_{0}$ to $p$, then the old length of the radial component $\gamma_{r}^{\prime}$ 
of $\gamma^{\prime}$ is simply the rate of change of $r$ along $\gamma$. Thus for the new length of $\gamma$ we have (parametrizing on $0 \leq t \leq 1$ )

$$
\begin{gathered}
\int_{0}^{1}\left\{\varphi\left(r^{2}(\gamma(t))\right)\left\langle\gamma^{\prime}(t), \gamma^{\prime}(t)\right\rangle\right\}^{1 / 2} d t \geq \int_{0}^{1}\left\{\varphi\left(r^{2}(\gamma(t))\right)\left\langle\gamma_{r}^{\prime}(t), \gamma_{r}^{\prime}(t)\right\rangle\right\}^{1 / 2} d t \\
=\int_{0}^{1} \varphi\left(r^{2}(\gamma(t))\right)^{1 / 2}\left|\frac{d r(\gamma(t))}{d t}\right| d t \geq \int_{0}^{r(p)} \varphi\left(r^{2}\right)^{1 / 2} d r
\end{gathered}
$$

But the last integral is the new length of the radial geodesic.

Corollary. Under the same hypothesis the spheres about $p_{0}$ are the same for either metric. If $P$ is a plane tangent at $p_{0}$, then the circle of radius $r$ tangent to $P$ is the curve with parameter $\theta: \exp r(x \cos \theta+y \sin \theta)$, where $(x, y)$ is an orthonormal basis of $P$. These circles are the same with respect to either metric (although their radii will be different).

Lemma 2. Besides the hypothesis of Lemma 1 we assume that

$$
\varphi(t)^{1 / 2}=1-c t+o\left(t^{2}\right) .
$$

If $K(p)$ is the sectional curvature of the plane section $P$ at $p_{0}$ with respect to the old metric, then the sectional curvature of $P$ with respect to the new metric is $K(P)+4 c$.

Proof. We use the fact that the circle of radius $r$ tangent to $P$ has circumference

$$
L(r)=2 \pi\left(r-K(P) r^{3} / 6+o\left(r^{4}\right)\right) .
$$

Let $s: M \rightarrow \boldsymbol{R}$ be the new distance from $p_{0}$. Then

$$
s=\int_{0}^{r} \varphi\left(t^{2}\right)^{1 / 2} d t=\int_{0}^{r}\left(1-c t^{2}+o\left(t^{4}\right)\right) d t=r-c r^{3} / 3+o\left(r^{5}\right) .
$$

Hence

$$
r=s+c r^{3} / 3+o\left(r^{5}\right)=s+c s^{3} / 3+o\left(s^{5}\right) .
$$

Let ' $L(s)$ be the new length of the circle of new radius $s$ tangent to $P$. Since the multiplier $\varphi\left(r^{2}\right)$ is constant along this circle, the circumferences are related by

$$
\begin{aligned}
{ }^{\prime} L(s) & =\varphi\left(r^{2}\right)^{1 / 2} L(r)=\left(1-c r^{2}+o\left(r^{4}\right)\right) 2 \pi\left(r-K(P) r^{3} / 6+o\left(r^{4}\right)\right) \\
& =2 \pi\left(r-[K(P)+6 c] r^{3} / 6+o\left(r^{4}\right)\right) \\
& =2 \pi\left(s+c s^{3} / 3-[K(P)+6 c] s^{3} / 6+o\left(s^{4}\right)\right) \\
& =2 \pi\left(s-[K(P)+4 c] s^{3} / 6+o\left(s^{4}\right)\right) .
\end{aligned}
$$


Corollary. Given a Riemannian manifold $M, p_{0} \in M, \varepsilon>0, c>0$, then there is a conformally equivalent metric such that

(a) the new metric coincides with the old one outside of an $\varepsilon$-ball about $p_{0}$,

(b) the sectional curvatures at $p_{0}$ are increased by $4 c$, and

(c) new distances are no greater than old ones and at least one-half as great as old ones.

Proof. Take $\varphi$ above so that $1 / 4<\varphi(t) \leq 1$ and $\varphi(t)=1$ when $t>\varepsilon^{2}$.

Nomizu and Ozeki [5] have proved that for every metric on a noncompact manifold there is a conformally equivalent complete (hence nonprolongable) metric and also there is a conformally equivalent bounded metric. We show that nonprolongability can be obtained without changing $C M$; part (b) of the theorem then follows from part (a) and the latter result of Nomizu and Ozeki.

Theorem 1. Let $M$ be a noncompact Riemannian manifold with metric $g$.

(a) There is a conformally equivalent nonprolongable metric such that Cauchy sequences are the same for both metrics.

(b) There is a bounded conformally equivalent nonprolongable metric.

Proof. By using the fact that $M$ has a countable dense subset and can be represented as a countable union of compact subsets, it is possible to construct a sequence $\{p(n)\}$ such that

(1) there are no convergent subsequences and

(2) given a divergent Cauchy sequence $\left\{q_{n}\right\}$ there is a subsequence $\{p(k(n))\}$ which is asymptotic to $\left\{q_{n}\right\}$.

Let $c_{n}$ be a sequence of positive numbers which diverges to $\infty$, and choose a sequence of $C^{\infty}$ functions $\varphi_{n}: \boldsymbol{R} \rightarrow(1 / 4,1]$ so that

(3) if all sectional curvatures at $p(n)$ are $\leq-2 c_{n}$, then $\varphi_{n}=1$;

(4) if some sectional curvature at $p(n)$ is $>-2 c_{n}$, then $\varphi_{n}(t)^{1 / 2}=$ $1-c_{n} t+o\left(t^{2}\right)$ and $\varphi_{n}(t)=1$ for $t>\varepsilon_{n}^{2}$, where $\varepsilon_{n}$ is a radius of a normal ball about $p(n)$ which does not meet the $\varepsilon_{k}$-ball about $p(k)$ for $k<n$. Let $r_{n}=$ distance from $p(n)$. Then $\varphi=\prod_{n=1}^{\infty} \varphi_{n}\left(r_{n}^{2}\right)$ is a $C^{\infty}$ function: $M \rightarrow(1 / 4,1]$.

The metric $\varphi g$ satisfies (a): because $(1 / 4) g<\varphi g \leq g$, they have the same Cauchy sequences; any divergent Cauchy sequence satisfies Proposition 1 (b) for the metric $\varphi g$.

\section{Riemannian completions}

A subset $C$ of a Riemannian manifold $M$ will be called extrinsically complete if the extrinsic distance function $d_{M} \mid C \times C$ is complete, that is, if all $d_{M^{-}}$ Cauchy sequences in $C$ converge in $C$. Thus extrinsically complete implies closed, and is equivalent to closed if $M$ is complete. A subset is compact if and only if it is extrinsically complete and bounded.

An arcwise connected subset $C$ of $M$ also has an intrinsic distance function $d_{C}$, where $d_{C}(p, q)$ is the infimum of lengths of curves in $C$ joining $p$ and $q$. 
Since the extrinsic distance function nowhere exceeds the intrinsic one, extrinsic completeness implies intrinsic completeness.

Morrow [3] has generalized the Nomizu-Ozeki completeness theorem by showing that the conformal alterations may be restricted to the complement of any compact subset $C$. We show they may be restricted to the complement of any extrinsically complete $C$. Of course, $C$ intrinsically complete would not do here. For example, on a flat torus take $C$ to be a dense geodesic and obtain $M$ by removing one point not on $C$; then the only metric on $M$ which agrees with the original on $C$ is the original, which is not complete.

Theorem 2. If $C$ is extrinsically complete in $M$, then $M$ admits a conformally equivalent complete metric agreeing with the original on $C$.

Proof. Let $B(p, a)$ denote the closed ball of radius $a$ at $p$. If the metric $g$ on $M$ is not already complete, $M$ carries the positive continuous function $r$ given by $r(p)=\sup \{a \mid B(p, a)$ is compact $\}$. Then the subset $K=$ $C \cup\left[\bigcup_{q \in B d r y C} B\left(q, \frac{1}{2} r(q)\right)\right]$, whose interior contains $C$, is itself extrinsically complete. In fact, suppose there is a Cauchy sequence $\left\{p_{i}\right\}$ in $K$ but not converging there. Since $\left\{p_{i}\right\}$ can have no subsequence in $C$, each $p_{i}$ may be assumed to lie in some $B\left(q_{i}, \frac{1}{2} r\left(q_{i}\right)\right)$. Then we have $r\left(p_{i}\right) \geq \frac{1}{2} r\left(q_{i}\right)$ and $r\left(p_{i}\right) \rightarrow 0$, hence also $r\left(q_{i}\right) \rightarrow 0$. This makes $\left\{p_{i}\right\}$ asymptotic to $\left\{q_{i}\right\}$ which lies in $C$, and gives a contradiction.

Now we may take $f \geq 1$ to be a smooth function on $M-C$ such that $f^{2} g$ is complete on each component of $M-C$. Let $K^{\prime}$ be a closed subset such that $C \subset$ interior $K^{\prime}$ and $K^{\prime} \subset$ interior $K$. Then there is a smooth partition of unity $\left\{h_{1}, h_{2}\right\}$ subordinate to the covering of $M$ by finterior $\left.K, M-K^{\prime}\right\}$. Let $d$ be the distance function for the metric $g$ on $M$ and ' $d$ the distance function for the metric $\left(h_{1}+h_{2} f\right)^{2} g$.

A'd-Cauchy sequence is also a $d$-Cauchy sequence, since $h_{1}+h_{2} f \geq 1$. Thus any ' $d$-sequence having infinitely many points in $K$ converges in $K$. If a ' $d$-sequence $\left\{p_{i}\right\}$ in $M-K$ does not eventually lie in a single component of $M-K$, then we may assume that $p_{i}$ and $p_{i+1}$ always lie in different components (by passing to a subsequence). Any curve from $p_{i}$ to $p_{i+1}$ passes through $K$, so taking a curve which is shorter than $2^{\prime} d\left(p_{i}, p_{i+1}\right)$ gives a point $q_{i}$ in $K$ such that ' $d\left(p_{i}, q_{i}\right)<2^{\prime} d\left(p_{i}, p_{i+1}\right)$. Then $\left\{q_{i}\right\}$ is a sequence ' $d$-asymptotic to $\left\{p_{i}\right\}$ and both converge.

Now suppose $\left\{p_{i}\right\}$ lies in a single component $M_{1}$ of $M-K$ which in turn is contained in a component $M_{2}$ of $M-C$. Note that $h_{1}=0$ on $M_{1}$, so that on $M_{1},\left(h_{1}+h_{2} f\right)^{2} g$ coincides with the metric $f^{2} g$. If the ' $d\left(p_{i}, p_{i+1}\right)$ can be approximated by lengths of curves which lie in $M_{1}$, then $\left\{p_{i}\right\}$ converges by the completeness of $f^{2} g$ on $M_{2}$. Otherwise curves whose lengths approximate ' $d\left(p_{i}, p_{i+1}\right)$ must run out of $M_{1}$ into $K$ and we get an asymptotic sequence in $K$ as above.

Call an extrinsic prolongation of $M$ a prolongation of $M$ to $N$ for which all $d_{N}$-Cauchy sequences in $M$ converge in $N$. This is in particular a prolongation 
on $\dot{M}$. Since for an extrinsic prolongation the closure of $M$ in $N$ is extrinsically complete in $N$, we immediately have:

Corollary. If a Riemannian manifold has an extrinsic prolongation, it has a Riemannian completion.

\section{References}

[ 1 ] S. Bochner, Fortsetzung Riemannscher Flächen, Math. Ann. 98 (1928) 406-421.

[2] E. Dubois, Beiträge zur Riemannschen Geometrie im Grossen, Comment. Math. Helvet. 41 (1966) 30-50.

[ 3 ] J. A. Morrow, The denseness of complete Riemannian metrics, J. Differential Geometry 4 (1970) 225-226.

[4] S. B. Myers, Riemannian manifolds in the large, Duke Math. J. 1 (1935) 39-49.

[5] K. Nomizu \& H. Ozeki, The existence of complete Riemannian metrics, Proc. Amer. Math. Soc. 12 (1961) 889-891.

[6] W. Rinow, Uber Zussammenhänge zwischen der Differential-geometrie im Grossen und im Kleinen, Math. Z. 35 (1932) 512-528.

UNIVERSITY OF ILLINOIS 